\title{
Fuzzy Similar Priority Method for Mixed Attributes
}

\author{
Jinshan Ma, ${ }^{1,2}$ Changsheng Ji, ${ }^{2}$ and Jing Sun ${ }^{3}$ \\ ${ }^{1}$ School of Energy Science and Engineering, Henan Polytechnic University, Jiaozuo, Henan 454000, China \\ ${ }^{2}$ School of Mines, China University of Mining and Technology, Xuzhou, Jiangsu 221116, China \\ ${ }^{3}$ Department of Financial Management, Jiaozuo Branch of Bank of China Ltd., Jiaozuo, Henan 454000, China
}

Correspondence should be addressed to Jinshan Ma; mjscumttf@163.com

Received 13 June 2014; Accepted 22 August 2014; Published 3 September 2014

Academic Editor: Olivier Bahn

Copyright (C) 2014 Jinshan Ma et al. This is an open access article distributed under the Creative Commons Attribution License, which permits unrestricted use, distribution, and reproduction in any medium, provided the original work is properly cited.

\begin{abstract}
Fuzzy similar priority ratio method is to select the most suitable one to the specific object from feasible alternatives. However, this method considering only the index values of real number has its disadvantages of inaccuracy in result and complexity in calculation. So, this method was extended to handle mixed attributes including real number, interval number, triangular fuzzy number, and trapezoidal fuzzy number. The proposed method decides the optimal alternative by the minimum of integrated nearness degrees calculated by all index vectors and the fixed index vectors based on the theory of similarity. The improved method can not only address mixed attributes but also simplify the calculation and improve the accuracy of result. A case study illustrated this method.
\end{abstract}

\section{Introduction}

Knowing some index values of the specific object, the most suitable one can be determined from some feasible alternatives using the method of fuzzy similar priority ratio, which is of fuzzy mathematic method [1]. Being of simple principle and clear meaning, this method has been widely used in many fields. However, the classical method only concerns the indices of real numbers. Furthermore, it has its disadvantages of inaccuracy in result and complexity in calculation. In decision making, the indices of an alternative may be characterized as interval number, triangular fuzzy number, and trapezoid fuzzy number. But the traditional method fails to deal with the mixed types of numbers. Article [2] presented a novel method in grey target decision making for handling mixed attributes. The method in [2] introduced the binary connection number provided in set pair analysis (SPA) $[3,4]$ to transform all types of indices into connection number vectors regarded as in the determinacy-uncertainty space $[5,6]$. Then all the nearness degrees of index vectors and the given index vectors could be calculated based on the theory of similarity. And the decision making was decided by the nearness degrees. The novel method addressed some alternatives for mixed attribute values, but its accuracy in solving the uncertain terms of index vectors needs improving.
Thus, an improved fuzzy similarity priority method is proposed to handle the mixed attributes based on the previous study of decision making by the nearness degrees. The improved fuzzy similar priority method has some features. First, it can deal with mixed attributes. Second, its calculation is simple compared with the traditional method. Third, it improves the accuracy of the nearness degrees provided in [2] through optimizing the uncertain terms of the index vectors.

The remainder of the organization is as follows. Section 2 presents some basic concepts. Section 3 discusses the proposed method. A case study is given in Section 4. And Section 5 concludes this work.

\section{Basic Concepts}

In this section, some concepts are presented.

Definition 1. Let $R$ be a set of real numbers; then $\widetilde{x}$ is called a fuzzy number if it has one of the following forms [6-11]:

(1) if $\tilde{x}=\left[x^{L}, x^{U}\right]$, then $\tilde{x}$ is called interval number, where $x^{L}, x^{U} \in R, x^{L}<x^{U}, x^{L}$, and $x^{U}$ are the interval number's lower limits and upper limits, respectively;

(2) if $\tilde{x}=\left[x^{L}, x^{M}, x^{U}\right]$, then $\tilde{x}$ is called triangular fuzzy number, where $x^{L}, x^{M}, x^{U} \in R, 0<x^{L}<x^{M}<$ 
$x^{U}, x^{L}$, and $x^{U}$ are the triangular fuzzy number's lower limits and upper limits, respectively, while $x^{M}$ is medium value;

(3) if $\tilde{x}=\left[x^{L}, x^{M}, x^{N}, x^{U}\right]$, then $\tilde{x}$ is called trapezoidal fuzzy number, where $0<x^{L}<x^{M}<x^{N}<x^{U} \in R$, $x^{L}$ and $x^{U}$ are the trapezoidal fuzzy number's lower limits and upper limits, respectively, while $\left[x^{M}, x^{N}\right]$ is medium interval.

Definition 2. Assume $\tilde{x}$ is a fuzzy number with its parameters which can be seen as $n(n \geqslant 2)$ observed values [2], and then the fuzzy number's average value, standard deviation, and maximum deviation are as follows:

$$
\begin{gathered}
\bar{x}=\frac{1}{n} \sum_{t=1}^{n} x_{t}, \\
s=\sqrt{\frac{1}{(n-1)} \sum_{t=1}^{n}\left(x_{t}-\bar{x}\right)^{2},} \\
m s=\max \left\{\left|x^{L}-\bar{x}\right|,\left|x^{U}-\bar{x}\right|\right\},
\end{gathered}
$$

where $x_{t}(t=1, \ldots, n)$ is the parameter of the fuzzy number, $\bar{x}$ is the average value of the parameters, $s$ is the standard deviation of the parameters, $m s$ is the maximum deviation of the parameters, and $x^{L}$ and $x^{U}$ are the fuzzy number's lower limits and upper limits, respectively.

Definition 3. Let $R$ be a set of real numbers; $A+B i$ is called binary connection number, where $A, B \in R, i \in[-1,1]$, $A$ denotes the deterministic term, $B$ denotes the uncertain term, and $i$ is a variable term unifying the determinacy and uncertainty of a set pair. So the determinacy-uncertainty vector based on connection number can be constructed as follows [2]:

$$
u(\bar{x}, v)=A+B i=\bar{x}+v i \quad(i \in[-1,1]),
$$

where $\bar{x}$ and $v$ are the concentrated value and the diverse value of $n$ parameter values of the same fuzzy number $\tilde{x}$, respectively, $\tilde{x}$ can be calculated using (1), and $v$ can be obtained by the following equation:

$$
v=\min \{s, m s\} \text {. }
$$

Article [12] provided the idea of using maximum deviation as the uncertain term of an index vector only involving the upper limits in trapezoidal number. However, considering both the lower limits and upper limits of a fuzzy number is more reasonable.

Remark 4. A real number can be regarded as the special form of a fuzzy number; thus the real number can also be converted into a binary connection number with the form $u(\bar{x}, v)=A+$ $0 i$, where $A$ is the real number itself and the uncertain term is 0 .

Definition 5. The mutual interaction of the average value $\bar{x}$ and the diverse value (standard deviation or maximum

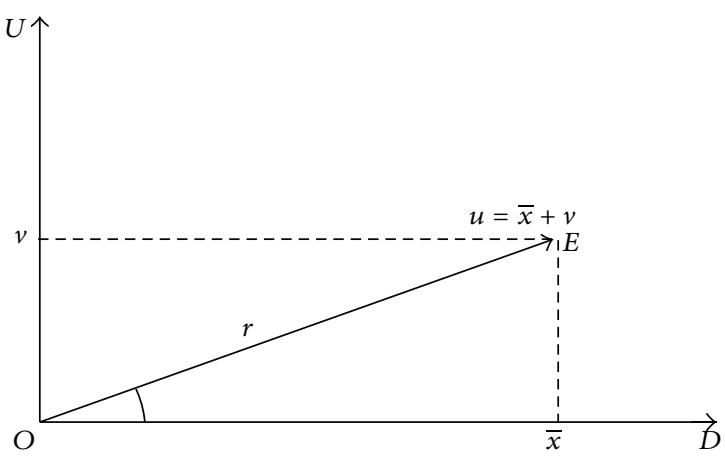

FIGURE 1: Determinacy-uncertainty space.

deviation) $v$ of the connection number $u(\bar{x}, v)$ can be mapped to the binary determinacy-uncertainty space ( $D-U$ space) based on SPA. If $u(\bar{x}, s)=\bar{x}+v i$ represents the vector of $D$ $U$ space, then the " $i$ " only denotes the signal of the uncertain term without representing the changeable value [2].

Figure 1 is a $D-U$ space. The $U$ axis and the $D$ axis represent the relative uncertain measure and the relative deterministic measure, respectively. In Figure 1, the $\bar{x}$ and the $v$ interact with each other, and the space reflection is the vector $\overline{O E}$ from $O$ to $E$, and the degree of interaction means the module of the vector $\overline{O E}$ denoted by $r$ [6]. Measures between vectors can use distance method [13] such as Hamming distance and Euclidean distance or similarity method [14] such as cosine value and projection value.

Definition 6. Let $X=\left(x_{1}, x_{2}, \ldots, x_{n}\right)$ be a vector; then

$$
|X|=\sqrt{\sum_{t=1}^{n} x_{t}^{2}}
$$

is called the module of $X$.

Definition 7. Let $X=\left(x_{1}, x_{2}, \ldots, x_{n}\right)$ and $Y=\left(y_{1}, y_{2}, \ldots, y_{n}\right)$ be two vectors; then

$$
\operatorname{Prj}_{Y}(X)=\frac{\sum_{t=1}^{n} x_{t} y_{t}}{\sqrt{\sum_{t=1}^{n} x_{t}^{2} \sum_{t=1}^{n} y_{t}^{2}}} \sqrt{\sum_{t=1}^{n} x_{t}^{2}}=\frac{\sum_{t=1}^{n} x_{t} y_{t}}{\sqrt{\sum_{t=1}^{n} y_{t}^{2}}}
$$

is the projection of vector $X$ on vector $Y$. Generally speaking, the bigger the value of $\operatorname{Prj}_{Y}(X)$ the more the similarity between $X$ and $Y[15]$.

Definition 8. Assume $r$ is the module of vector $Y$ and $p_{r j}$ is the projection value of vector $X$ on vector $Y$; then

$$
d_{n 0}=\left|r-p_{r j}\right|
$$

is the distance of projection value $p_{r j}$ and the module $r$ is called nearness degree. When the two index vectors are all transformed from real numbers, the $d_{n 0}$ in (8) is actually degraded to Hamming distance [2]. 
Remark 9. Measuring the similarity of the two index vectors with nearness degree has its advantages over cosine value and projection value. First, it can specifically reflect the similarity of the two vectors whether they transformed from real number or fuzzy number. Second, it is the generalized distance especially for that it is degraded to Hamming distance when the two index vectors are converted from real numbers. The detailed discussion can be seen in the paper [2].

\section{Fuzzy Similar Priority Method for Mixed Attributes}

\subsection{Brief Introduction to the Method of Fuzzy Similar Priority Ratio}

\subsubsection{Fuzzy Preference Relation}

Definition 10. Let $G=\left(g_{1}, g_{2}, \ldots, g_{m}\right)$ be a set of alternatives; if $g_{i}$ and $g_{j}(i, j=1,2, \ldots, m)$ are compared with the alternative $g_{0}$, then the fuzzy preference relation $R$ is described as follows.

$R=\left(r_{i j}\right)_{m \times m}, r_{i j} \in[0,1](i, j=1,2, \ldots, m) R: A \times A \rightarrow$ $[0,1]$, and $r_{i j}$ satisfies the following relations.

(1) $r_{i i}=0, i=1,2, \ldots, m ;(2) r_{i j}+r_{j i}=1(i \neq j, i, j=$ $1,2, \ldots, m)$.

Equation (1) indicates that $g_{i}$ has indifference with itself, so $r_{i i}=0$. And (2) indicates that if $g_{i}$ is preferred to $g_{j}$, denoted by $r_{i j}$, then $g_{j}$ is preferred to $g_{i}$, denoted by $r_{j_{i}}=$ $1-r_{i j}$, especially, and $r_{i j}=1$ indicates that $g_{i}$ is absolutely preferred to $g_{j}$ with respect to $g_{0}[16-18]$.

3.1.2. Algorithm of Fuzzy Similar Priority Ratio. Let $X_{0}=$ $\left(x_{01}, x_{02}, \ldots, x_{0 n}\right)$ be index vector of the specific object and let the index value of the $i$ th feasible alternative be $x_{i j}(i=$ $1,2, \ldots, m, j=1,2, \ldots, n)$, so the algorithm of fuzzy similar priority ratio is as follows $[16,19]$.

Step 1. With respect to the attribute $A_{k}$, calculate the Hamming distances of $x_{i k}$ and $x_{j k}$ with $x_{0 k}$, respectively,

$$
d_{i k}=\left|x_{i k}-x_{0 k}\right|, \quad d_{j k}=\left|x_{i j k}-x_{0 k}\right| .
$$

Step 2. Construct the fuzzy preference relation

$$
r_{i j}=\frac{d_{j k}}{d_{i k}+d_{j k}}
$$

so the fuzzy similar matrix for attribute $A_{k}$ is

$$
R^{k}=\left(\begin{array}{ccc}
r_{11} & \ldots & r_{1 m} \\
\vdots & \ddots & \vdots \\
r_{m 1} & \cdots & r_{m m}
\end{array}\right), \quad k=1,2, \ldots, n .
$$

Step 3. A series of $\lambda$-sectional sets can be determined through operating $R^{k}$ with the $\lambda$ ascending in $[0,1]$. To get the $\lambda$ sectional set, the elements of $R^{k}$ must be compared with the $\lambda$. The elements of $R^{k}$ will be rewritten as 1 if they are no less than the $\lambda$ value. Under attribute $A_{k}$, the most similarity alternative to the specific object is the one whose row first gets all " 1 " except the diagonal elements and the alternative acquires the order value "1." Then, the impact of the alternative must be eliminated by removing the row corresponding to the alternative and the same column in $R^{k}$. Repeating the same work, a series of similarity alternatives can be obtained and assigned the order values $2,3, \ldots, m$. And the order value that the alternative gains under attribute $A_{k}$ is denoted by $C_{i k}(i=1,2, \ldots, m, k=1,2, \ldots, n)$.

Step 4. Integrate the order values under different attributes for every alternative denoted by $\mathrm{TC}_{i}$ with the following equation:

$$
\mathrm{TC}_{i}=\sum_{k=1}^{n} C_{i k}, \quad i=1,2, \ldots, m, k=1,2, \ldots, n .
$$

Step 5. Rank the alternatives with $\mathrm{TC}_{i}$ in ascending order, and the optimal alternative to the specific object is the one with the minimum value $\mathrm{TC}_{i}$.

\subsection{Fuzzy Similar Priority Method for Mixed Attributes}

3.2.1. Methodology of the Proposed Method. Let $X_{0}=\left(x_{01}\right.$, $\left.x_{02}, \ldots, x_{0 j}, \ldots, x_{0 n}\right)$ be index vector of the specific object and let the index value of the $i$ th feasible alternative be $x_{i j}(i=$ $1,2, \ldots, m, j=1,2, \ldots, n)$, where $x_{i j}$ and $x_{0 j}$ can be expressed as fuzzy numbers or real numbers. The algorithm of the improved fuzzy similar priority method can be concluded as follows [20].

Step 1. Calculate the average values, standard deviations, and maximum deviations of all indices $\left(x_{i j}\right.$ and $\left.x_{0 j}\right)$ by using Equations from (1) to (3).

Step 2. Translate all index values into the binary $A+B i$ connection number vectors using (4) and (5). So the converted index vectors can be expressed as

$$
u_{s t}=\bar{x}_{s t}+v_{s t} i \quad(i \in[-1,1]), s=0, \ldots, m, t=1, \ldots, n .
$$

Step 3. Calculate the nearness degrees of all index vectors $u_{s t}(s=1, \ldots, m, t=1, \ldots, n)$ and the fixed index vectors $u_{s t}(s=0, t=1, \ldots, n)$ denoted by $q_{i j}(i=1, \ldots, m, j=$ $1, \ldots, n)$ using Equations from (6) to (8) and (13).

Step 4. Normalize the nearness degrees of all index vectors and the fixed index vector by

$$
z_{i j}=\frac{q_{i j}}{\sum_{i=1}^{m} q_{i j}}, \quad i=1, \ldots, m ; j=1, \ldots, n,
$$

where $z_{i j}$ is the normalized nearness degree.

Step 5. Determine the weights of all attributes denoted as $\omega_{j}(j=1, \ldots, n)$ by objective method or subjective method.

Step 6. Integrate the normalized nearness degrees considering weights of all attributes using the following equation:

$$
w_{i}=\omega_{j} z_{i j}, \quad i=1, \ldots, m, j=1, \ldots, n .
$$


TABLE 1: Safety data from coal mines.

\begin{tabular}{lccccc}
\hline$S_{i}$ & $A_{1}$ & $A_{2}$ & $A_{3}$ & $A_{4}$ & $A_{5}$ \\
\hline$S_{1}$ & {$[18,20,25]$} & {$[4,6,8]$} & {$[180,260]$} & 12 & {$[90,91,93,94]$} \\
$S_{2}$ & {$[12,16,20]$} & {$[2.8,4,4.3]$} & {$[190,210]$} & 6 & {$[88,89,91,92]$} \\
$S_{3}$ & {$[22,25,31]$} & {$[8.8,9.2,9.6]$} & {$[160,200]$} & 10 & {$[84,87,89,92]$} \\
$S_{4}$ & {$[6,11,13]$} & {$[3.6,4,4.4]$} & {$[240,280]$} & 8 & {$[91,93,95,97]$} \\
$S_{5}$ & {$[24,32,34]$} & {$[7.3,8.3,9]$} & {$[340,360]$} & 10 & {$[94,95,97,98]$} \\
$S_{6}$ & {$[14,16,18]$} & {$[9.4,9.6,9.8]$} & {$[360,440]$} & 6 & {$[82,84,88,90]$} \\
$S_{7}$ & {$[33,42,45]$} & {$[13,14,15]$} & {$[550,650]$} & 6 & {$[.2$} \\
$S_{8}$ & {$[9,12,15]$} & {$[12,13,13.4]$} & {$[100,140]$} & 10 & 3.6 \\
$S_{0}$ & {$[8,12,22]$} & {$[5.2,6,6.2]$} & {$[150,160]$} & 12 & {$[89,94,96,97]$} \\
\hline
\end{tabular}

Step 7. Rank the feasible alternatives by the integrated normalized nearness degrees $w_{i}(i=1, \ldots, m)$.

3.2.2. Merits of the Proposed Method. The improved fuzzy similar method has its advantages over the traditional method. Above all, the proposed method can address the mixed numbers based on the similarity of the improved determinacy-uncertainty index vectors through optimizing the uncertain terms of them. Moreover, it can simplify the calculation by avoiding the traditional tedious computing procedure such that Steps 2 and 3 in the traditional method are reduced or avoided. Nevertheless, it determines the optimal alternative by the minimum of integrated nearness degrees instead of the minimum order values and can improve the accuracy of results. Finally, it can also reflect the decision makers' preferences by considering attribute weights.

\section{Cases Study}

4.1. Background. A coal enterprise is to select suitable safety management model from some coal enterprise with different advanced safety management level. The safety management model of a coal enterprise depends on some indices including seam dip $\left({ }^{\circ}\right)$, methane emission rate $\left(\mathrm{m}^{3} / \mathrm{t}\right)$, water inflow $\left(\mathrm{m}^{3} / \mathrm{h}\right)$, spontaneous combustion period (month), ventilating structures qualification rate $(\%)$, and equivalent orifice $\left(\mathrm{m}^{2}\right)$ $[21,22]$ denoted by $A_{1}$ to $A_{6}$ respectively. Thus some coal enterprises with different index values can be regarded as different alternatives. Here $S_{0}$ represents the specific coal enterprise wants to learn advanced safety management experience. And the eight feasible alternatives (coal enterprises) are denoted as $S_{1}$ to $S_{8}$ respectively. Besides the index values are given as mixed attribute values including real numbers, interval numbers, triangle fuzzy numbers and trapezoid fuzzy numbers. The aim of the decision makers is to seek for the most suitable enterprise whose safety management experiece can be learnt. So the proposed method can address this problem easily. The data is shown in Table 1.

\subsection{Process of Decision Making}

4.2.1. Calculate the Parameters of Index Vectors. All average values, standard deviations, and maximum deviations of all indices can be calculated using Equations from (1) to (3) and the results are shown in Table 2.

4.2.2. Translate All Index Values into Connection Number Vectors. Having obtained the deterministic terms and uncertain terms of the connection numbers above, all index values can be transformed into index vectors using (4). Table 3 shows the connection numbers converted from all indices.

4.2.3. Calculate Nearness Degrees of All Index Vectors with the Fixed Index Vectors. All nearness degrees of all index vectors with the fixed index vectors can be calculated as shown in Table 4 using Equations from (6) to (8).

4.2.4. Normalize the Nearness Degrees. The normalized nearness degrees shown in Table 5 can be calculated using (14).

4.2.5. Integrate the Normalized Nearness Degrees. Without considering all attribute weights, the integrated nearness degrees of all alternatives can be calculated as $W=$ (0.272792, 0.427914, 0.610936, 0.666333, 1.191736, 0.754849, and 1.5579080 .517532 ). So the alternatives ranking is $S_{1}>$ $S_{2}>S_{8}>S_{3}>S_{4}>S_{6}>S_{5}>S_{7}$. In brief, $S_{1}$ is the best selection for $S_{0}$.

If the attributes $\omega=(0.11,0.26,0.07,0.12,0.21,0.23)$ are given, then the weighted integrated nearness degrees of all alternatives can be calculated as $W=(0.042693,0.069455$, 0.112996, 0.110897, 0.210185, 0.116780, 0.232191, and 0.104803). So the alternatives ranking is $S_{1}>S_{2}>S_{8}>S_{4}>S_{3}>S_{6}>$ $S_{5}>S_{7}$.

4.3. Discussion. A comparison between the order value method and the nearness degree method is performed to illustrate the advantage of the proposed method to determine the optimal alternative. Table 6 shows the order values of all indices near the fixed indices determined by nearness degrees. The order values of all alternatives under some attribute are given by their index nearness degrees in terms of the principle of the smaller the better.

So the total integrated order values can be calculated as $\mathbf{W}$ $=(16,16,21,26,34,27,39,19)$ using (12). And the alternatives ranking is $S_{1}=S_{2}>S_{8}>S_{3}>S_{4}>S_{6}>S_{5}>S_{7}$. 
TABLE 2: Average values, standard deviations, and maximum deviations of all indices.

\begin{tabular}{lccccc}
\hline$S_{i}$ & $A_{1}$ & $A_{2}$ & $A_{3}$ & $A_{4}$ & $A_{5}$ \\
\hline$S_{1}$ & $21 / 3.605551 / 4$ & $6 / 2 / 2$ & $220 / 56.56854 / 40$ & $12 / 0 / 0$ & $92 / 1.825742 / 2$ \\
$S_{2}$ & $16 / 4 / 4$ & $3.7 / 0.793725 / 0.9$ & $200 / 14.14214 / 10$ & $6 / 0 / 0$ & $90 / 1.825742 / 2$ \\
$S_{3}$ & $26 / 4.582576 / 5$ & $9.2 / 0.4 / 0.4$ & $180 / 28.28427 / 20$ & $10 / 0 / 0$ & $88 / 3.366502 / 4$ \\
$S_{4}$ & $10 / 3.605551 / 4$ & $4 / 0.4 / 0.4$ & $260 / 28.28427 / 20$ & $8 / 0 / 0$ & $94 / 2.581989 / 3$ \\
$S_{5}$ & $30 / 5.291503 / 6$ & $8.2 / 0.8544 / 0.9$ & $350 / 14.14214 / 10$ & $10 / 0 / 0$ & $96 / 1.825742 / 2$ \\
$S_{6}$ & $16 / 2 / 2$ & $9.6 / 0.2 / 0.2$ & $400 / 56.56854 / 40$ & $6 / 0 / 0$ & $86 / 3.651484 / 4$ \\
$S_{7}$ & $40 / 6.244998 / 7$ & $14 / 1 / 1$ & $600 / 70.71068 / 50$ & $6 / 0 / 0$ & $95 / 1.825742 / 2$ \\
$S_{8}$ & $12 / 3 / 3$ & $12.8 / 0.72111 / 0.6$ & $120 / 28.28427 / 20$ & $10 / 0 / 0$ & $91 / 2.160247 / 3$ \\
$S_{0}$ & $14 / 7.211103 / 8$ & $5.8 / 0.52915 / 0.6$ & $155 / 7.071068 / 5$ & $12 / 0 / 0$ & $89 / 0$ \\
\hline
\end{tabular}

Note: Each term with the form " $a / b / c$ " in Table 2 denotes "average value/standard deviation/maximum deviation", for example the term "21/3.605551/4" represents $a=21, b=3.605551$, and $c=4$.

TABLE 3: Index vectors transformed from index values.

\begin{tabular}{|c|c|c|c|c|c|c|}
\hline$S_{i}$ & $A_{1}$ & $A_{2}$ & $A_{3}$ & $A_{4}$ & $A_{5}$ & $A_{6}$ \\
\hline$S_{1}$ & $21+3.605551 i$ & $6+2 i$ & $220+40 i$ & $12+0 i$ & $92+1.825742 i$ & $1.8+0 i$ \\
\hline$S_{2}$ & $16+4 i$ & $3.7+0.793725 i$ & $200+10 i$ & $6+0 i$ & $90+1.825742 i$ & $1.4+0 i$ \\
\hline$S_{3}$ & $26+4.582576 i$ & $9.2+0.4 i$ & $180+20 i$ & $10+0 i$ & $88+3.366502 i$ & $2.7+0 i$ \\
\hline$S_{4}$ & $10+3.605551 i$ & $4+0.4 i$ & $260+20 i$ & $8+0 i$ & $94+2.581989 i$ & $1.2+0 i$ \\
\hline$S_{5}$ & $30+5.291503 i$ & $8.2+0.8544 i$ & $350+10 i$ & $10+0 i$ & $96+1.82574 i$ & $3.6+0 i$ \\
\hline$S_{6}$ & $16+2 i$ & $9.6+0.2 i$ & $400+40 i$ & $6+0 i$ & $86+3.651484 i$ & $1.3+0 i$ \\
\hline$S_{7}$ & $40+6.244998 i$ & $14+1 i$ & $600+50 i$ & $6+0 i$ & $95+1.825742 i$ & $2.1+0 i$ \\
\hline$S_{8}$ & $12+3 i$ & $12.8+0.6 i$ & $120+20 i$ & $10+0 i$ & $91+2.160247 i$ & $1.5+0 i$ \\
\hline$S_{0}$ & $14+7.211103 i$ & $5.8+0.52915 i$ & $155+5 i$ & $12+0 i$ & $89+1.825742 i$ & $1.7+0 i$ \\
\hline
\end{tabular}

TABLE 4: Nearness degrees of all index vectors with the fixed index vectors.

\begin{tabular}{cccccc}
\hline$q_{i j}$ & $A_{1}$ & $A_{2}$ & $A_{3}$ & $A_{4}$ & $A_{5}$ \\
\hline$q_{1 j}$ & 4.5720046 & 0.332808 & 66.094653 & 0 & 2.999369 \\
$q_{2 j}$ & 0.3076204 & 2.067276 & 45.137812 & 6 & 0.99979 \\
$q_{3 j}$ & 9.4643938 & 3.374204 & 25.470622 & 2 & 0.968189 \\
$q_{4 j}$ & 5.207005 & 1.804289 & 105.42903 & 4 & 5.014459 \\
$q_{5 j}$ & 13.345019 & 2.419625 & 195.05983 & 2 & 6.998528 \\
$q_{6 j}$ & 0.6081906 & 3.754378 & 246.00107 & 6 & 2.961924 \\
$q_{7 j}$ & 22.671639 & 8.208865 & 446.21951 & 6 & 5.998738 \\
$q_{8 j}$ & 3.7062887 & 6.977486 & 34.498185 & 2 & 0.5 \\
\hline
\end{tabular}

TABLE 5: Normalized nearness degrees of all index vectors with the fixed index vectors.

\begin{tabular}{lcccccc}
\hline$Z_{i j}$ & $A_{1}$ & $A_{2}$ & $A_{3}$ & $A_{4}$ & $A_{5}$ \\
\hline$Z_{1 j}$ & 0.076350 & 0.0115 & 0.0567867 & 0 & 0.107322 & 0.020833 \\
$Z_{2 j}$ & 0.005137 & 0.071436 & 0.0387812 & 0.2143 & 0.035774 & 0.0625 \\
$Z_{3 j}$ & 0.158050 & 0.116597 & 0.0218837 & 0.0714 & 0.034643 \\
$Z_{4 j}$ & 0.086954 & 0.062348 & 0.0905817 & 0.1429 & 0.179425 & 0.208333 \\
$Z_{5 j}$ & 0.222855 & 0.083611 & 0.16759 & 0.0714 & 0.250418 & 0.104167 \\
$Z_{6 j}$ & 0.010156 & 0.129735 & 0.2113573 & 0.2143 & 0.105982 & 0.395833 \\
$Z_{7 j}$ & 0.378604 & 0.283662 & 0.3833795 & 0.2143 & 0.214644 & 0.083333 \\
$Z_{8 j}$ & 0.061893 & 0.241111 & 0.0296399 & 0.0714 & 0.071793 & 0.041667 \\
\hline
\end{tabular}


TABLE 6: Order values of index vectors with fixed index vectors.

\begin{tabular}{|c|c|c|c|c|c|c|c|}
\hline$C_{i j}$ & $A_{1}$ & $A_{2}$ & $A_{3}$ & $A_{4}$ & $A_{5}$ & $A_{6}$ & $\overline{\sum C_{i j}}$ \\
\hline$\overline{C_{1 j}}$ & 4 & 1 & 4 & 1 & 5 & 1 & 16 \\
\hline$C_{2 j}$ & 1 & 3 & 3 & 4 & 2 & 3 & 16 \\
\hline$C_{3 j}$ & 6 & 5 & 1 & 2 & 1 & 6 & 21 \\
\hline$C_{4 j}$ & 5 & 2 & 5 & 3 & 6 & 5 & 26 \\
\hline$C_{5 j}$ & 7 & 4 & 6 & 2 & 8 & 7 & 34 \\
\hline$C_{6 j}$ & 2 & 6 & 7 & 4 & 4 & 4 & 27 \\
\hline$C_{7 j}$ & 8 & 8 & 8 & 4 & 7 & 4 & 39 \\
\hline$C_{8 j}$ & 3 & 7 & 2 & 2 & 3 & 2 & 19 \\
\hline
\end{tabular}

TABLE 7: Alternatives ranking with two types of method.

\begin{tabular}{|c|c|c|c|c|c|c|}
\hline \multirow{2}{*}{$S_{i}$} & \multicolumn{2}{|c|}{ Order values } & \multicolumn{2}{|c|}{ Nearness degrees (no weights) } & \multicolumn{2}{|c|}{ Nearness degrees (weights) } \\
\hline & $C_{i}$ & Ranking & $W_{i}$ & Ranking & $W_{i}$ & Ranking \\
\hline$S_{1}$ & 16 & 1 & 0.272792 & 1 & 0.042693 & 1 \\
\hline$S_{2}$ & 16 & 1 & 0.427914 & 2 & 0.069455 & 2 \\
\hline$S_{3}$ & 21 & 3 & 0.610936 & 4 & 0.112996 & 5 \\
\hline$S_{4}$ & 26 & 4 & 0.666333 & 5 & 0.110897 & 4 \\
\hline$S_{5}$ & 34 & 6 & 1.191736 & 7 & 0.210185 & 7 \\
\hline$S_{6}$ & 27 & 5 & 0.754849 & 6 & 0.116780 & 6 \\
\hline$S_{7}$ & 39 & 7 & 1.557908 & 8 & 0.232191 & 8 \\
\hline$S_{8}$ & 19 & 2 & 0.517532 & 3 & 0.104803 & 3 \\
\hline
\end{tabular}

Table 7 is the comparison of two types of calculation method: one by integrated order values and one by integrated nearness degrees. However, decision making by integrated nearness degrees includes considering the attribute weights or not.

Seen from Table 7, alternatives ranking by order values is rough with the alternatives $S_{1}$ and $S_{2}$ in the same position. While the results by nearness degrees are accuracy with the alternatives $S_{1}$ and $S_{2}$ in different position. Furthermore, the proposed method can also use the attribute weights to reflect the decision makers' preferences. There is somewhat difference between the decision making considering attribute weights or not.

\section{Conclusions}

An improved fuzzy similar priority method was proposed to handle alternatives for mixed attributes. The proposed method solves the mixed attributes using the nearness degrees as derived from the similarity of the index vectors. And it improves the uncertain term of the index vector by selecting the minimum of the standard deviation or the minimum deviation of the parameters of the index and will contribute to the accuracy in result. Furthermore, the new method can reflect the decision maker's preferences through considering attribute weights. Another feature of this method is that its procedure and calculation are simple. A case study illustrated that it has advantages over the classical method.

\section{Conflict of Interests}

The authors declare that there is no conflict of interests regarding the publication of this paper.

\section{Acknowledgments}

The authors thank the Key Research Project of Science and Technology of Henan province (Grant no. 13B620033), Natural Science Foundation of the Education Department of Henan Province (Grant no. 2011B620001), and Henan Coal Mine Safety Production Technology Development Project (Grant no.H09-50) for the support.

\section{References}

[1] L. A. Zadeh, "Fuzzy sets," Information and Computation, vol. 8, pp. 338-353, 1965.

[2] J. S. Ma and C. S. Ji, "Generalized grey target decision method for mixed attributes based on connection number," Journal of Applied Mathematics, vol. 2014, Article ID 763543, 8 pages, 2014.

[3] K. Q. Zhao and A. L. Xuan, "Set pair theory-a new theory method of non-define and its applications," Systems Engineering, vol. 14, no. 1, pp. 18-23, 1996.

[4] K. Q. Zhao, Set Pair Analysis and Its Prelimiary Application, Zhejiang Press of Science and Technology, Hangzhou, China, 2000.

[5] K. Q. Zhao, "The theoretical basis and basic algorithm of binary connection $\mathrm{A}+\mathrm{Bi}$ and its application in AI," CAA I Transactions on Intelligent Systems, vol. 3, no. 6, pp. 476-486, 2008. 
[6] K. Q. Zhao, "Decision making algorithm based on set pair analysis for use when facing multiple uncertain in attributes," CAAI Transactions on Intelligent Systems, vol. 5, no. 1, pp. 41-50, 2010.

[7] T.-P. Lo and S.-J. Guo, "Effective weighting model based on the maximum deviation with uncertain information," Expert Systems with Applications, vol. 37, no. 12, pp. 8445-8449, 2010.

[8] Z. Yue, "An extended TOPSIS for determining weights of decision makers with interval numbers," Knowledge-Based Systems, vol. 24, no. 1, pp. 146-153, 2011.

[9] L. Liu, J.-H. Chen, G.-M. Wang, and D.-Z. Lao, "Multiattributed decision making for mining methods based on grey system and interval numbers," Journal of Central South University, vol. 20, no. 4, pp. 1029-1033, 2013.

[10] A. I. Ban and L. Coroianu, "Nearest interval, triangular and trapezoidal approximation of a fuzzy number preserving ambiguity," International Journal of Approximate Reasoning, vol. 53, no. 5, pp. 805-836, 2012.

[11] D. Luo and X. Wang, "The multi-attribute grey target decision method for attribute value within three-parameter interval grey number," Applied Mathematical Modelling, vol. 36, no. 5, pp. 1957-1963, 2012.

[12] W. X. Wu, "Utilization of the interval number in multi-attribute decision-making of trapezoidal fuzzy numbers," Mathematics in Practice and Theory, vol. 43, no. 1, pp. 160-166, 2013.

[13] Z. Xu and M. Xia, "Distance and similarity measures for hesitant fuzzy sets," Information Sciences, vol. 181, no. 11, pp. 2128-2138, 2011.

[14] J. Ye, "Cosine similarity measures for intuitionistic fuzzy sets and their applications," Mathematical and Computer Modelling, vol. 53, no. 1-2, pp. 91-97, 2011.

[15] G. W. Wei, Theory and Methods of Multiple Attribute Decision Making Based on Fuzzy Information, China Economic Publishing House, Beijing, China, 2010.

[16] H. Q. He, K. B. Wen, Z. X. Chen, and W. J. Ye, "Case-based reasoning and fuzzy analogy preferred ratio for effective depth of collapsible loess treated with dynamic consolidation," Journal of Engineering Geology, vol. 17, no. 11, pp. 88-93, 2009.

[17] Y. L. Jiang, "An approach to group decision making based on interval fuzzy preference relations," Journal of Systems Science and Systems Engineering, vol. 16, no. 1, pp. 113-120, 2007.

[18] Y. Xu, Q. Da, and L. Liu, "Normalizing rank aggregation method for priority of a fuzzy preference relation and its effectiveness," International Journal of Approximate Reasoning, vol. 50, no. 8, pp. 1287-1297, 2009.

[19] S. H. Wang, "Forecasting the bid of Engineering projects with fuzzy similar-priority comparison," Journal of Southwest Jiao Tong University, vol. 36, no. 1, pp. 96-99, 2001.

[20] J. Y. Zhou, S. D. Song, and Z. F. Yuan, "Method of synthetic distance priority in fuzzy decesion," Acta Universitatis Agriculturalis Boreali-Occidentalis Acta Universitatis Agriculturalis Boreali-Occidentalis, vol. 24, no. 2, pp. 71-74, 1996.

[21] J. S. Ma, "Grey target decision method for a variable target centre based on the decision maker's preferences," Journal of Applied Mathematics, vol. 2014, Article ID 572529, 6 pages, 2014.

[22] S. G. Cao, A. M. Xu, Y. B. Liu, and L. Q. Zhang, "Comprehensive assessment of security in coal mines based on grey relevance analysis," Journal of Mining \& Safety Engineering, vol. 24, no. 2, pp. 141-145, 2007. 


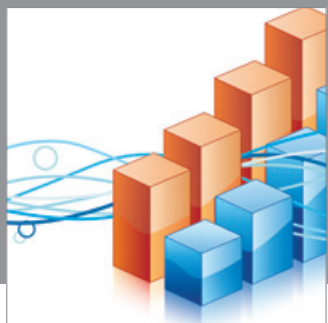

Advances in

Operations Research

mansans

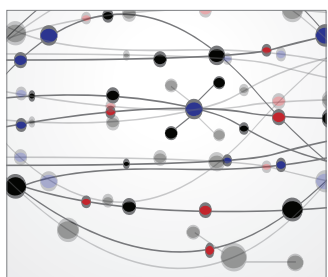

The Scientific World Journal
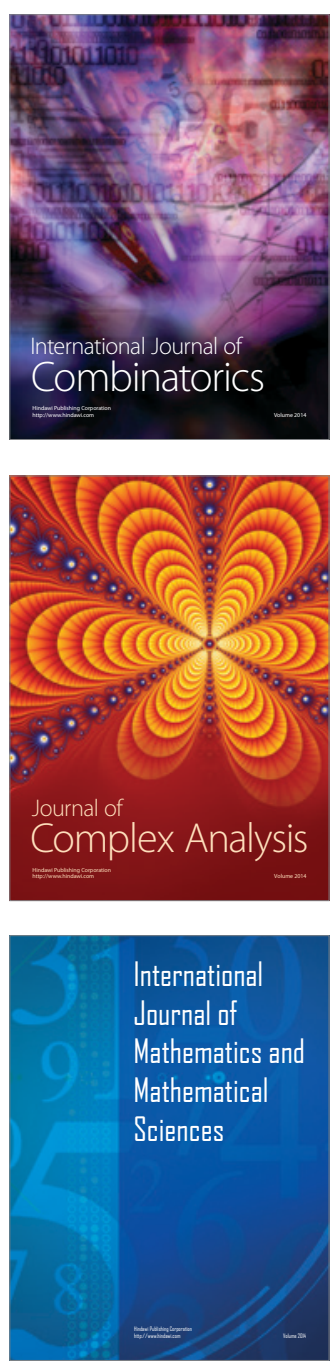
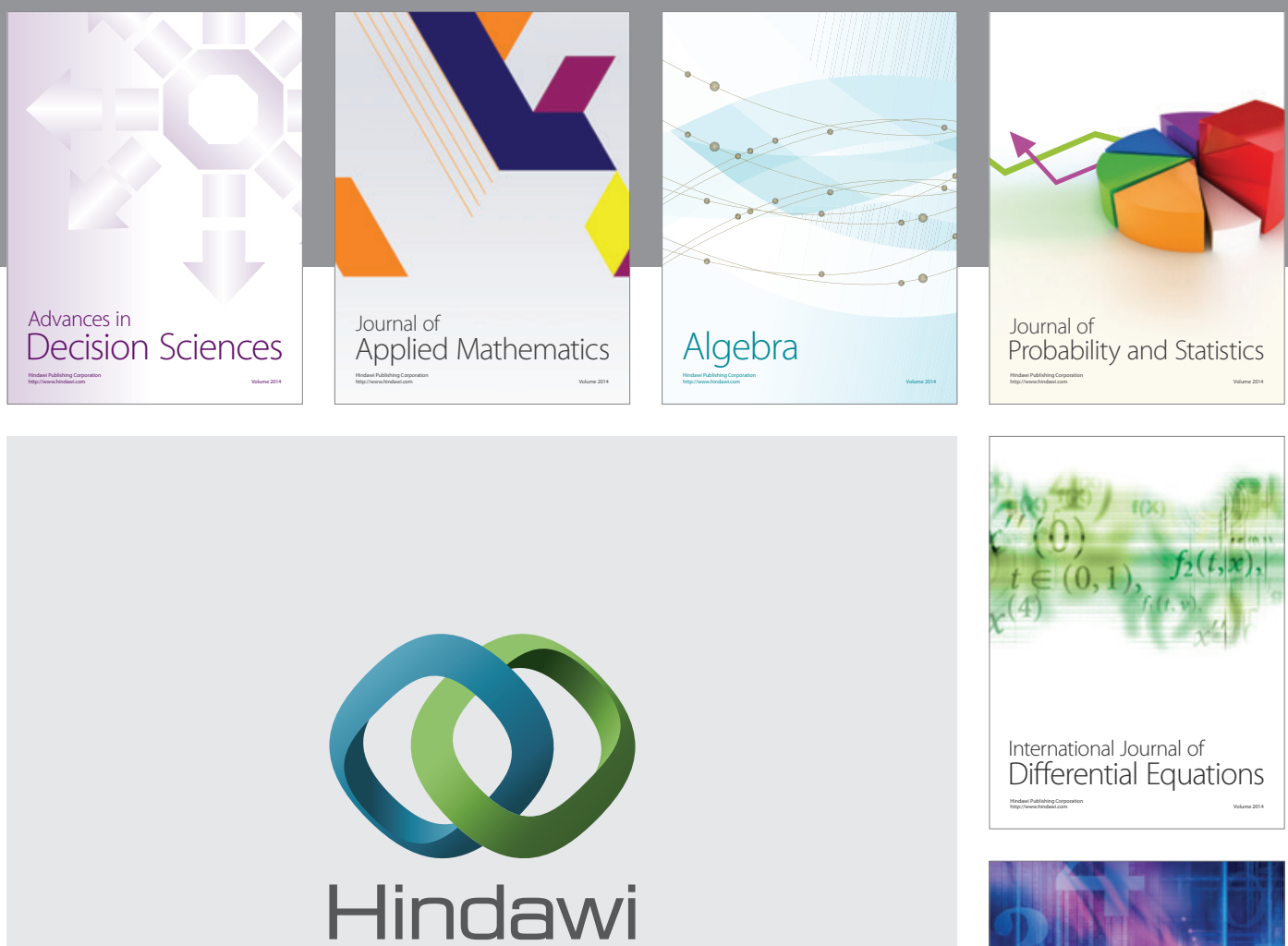

Submit your manuscripts at http://www.hindawi.com
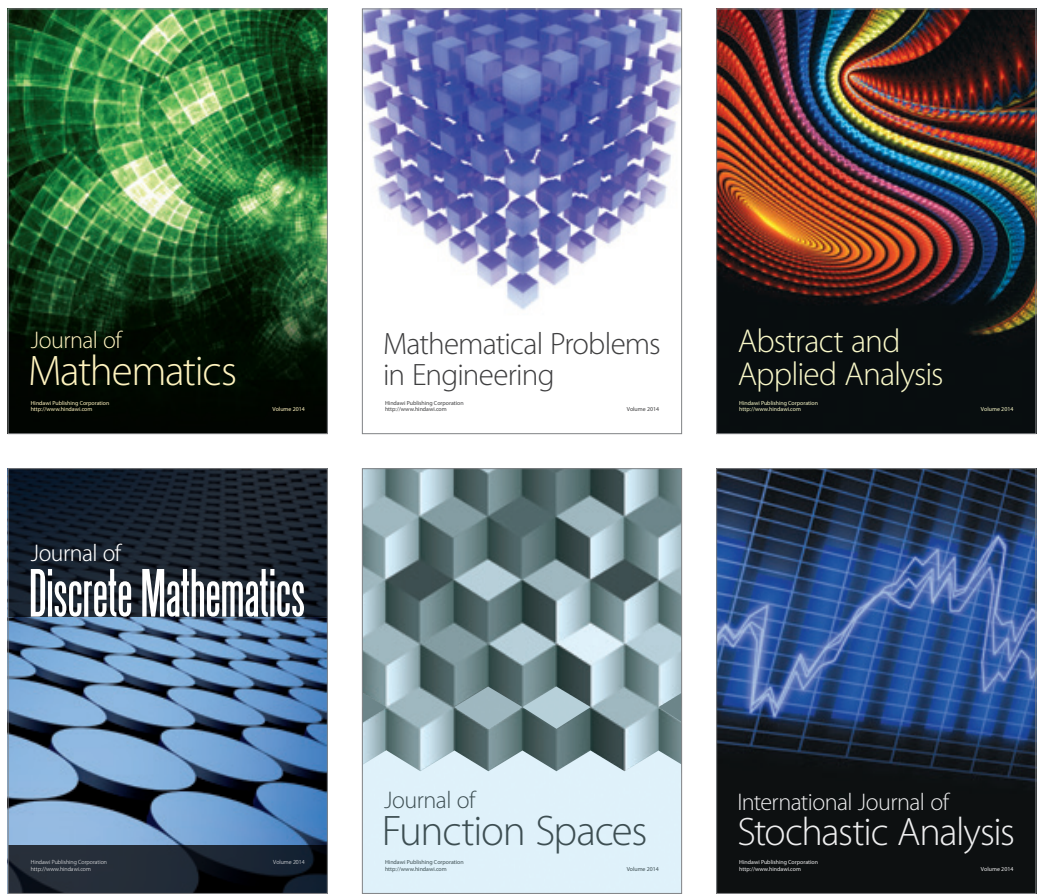

Journal of

Function Spaces

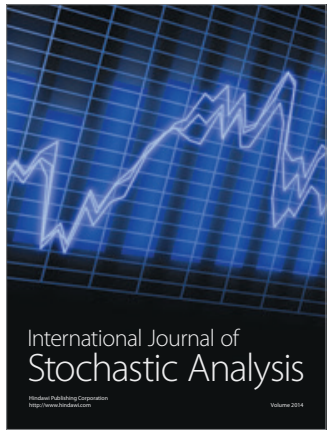

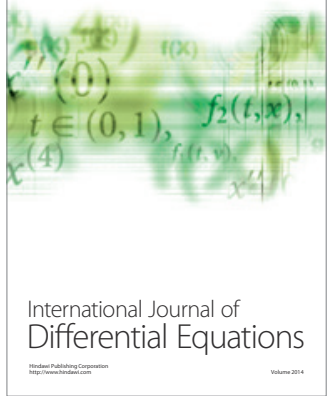
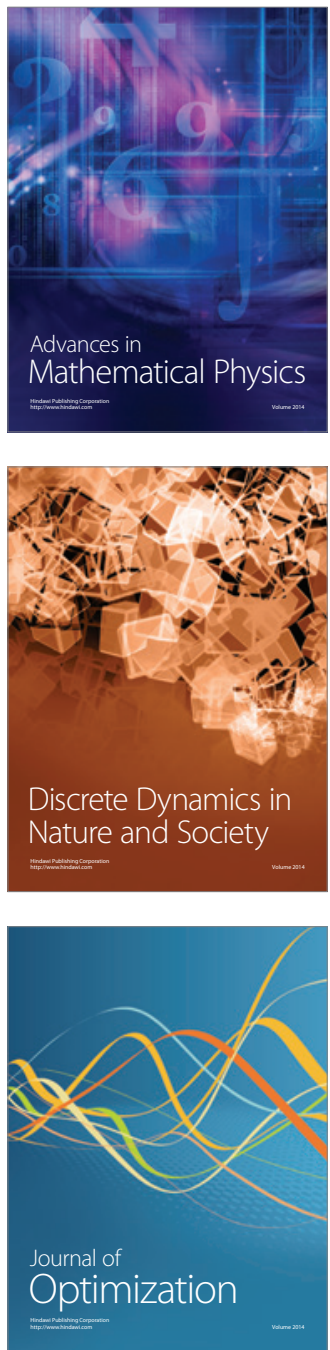